Teknokultura. Revista de Cultura Digital y Movimientos Sociales

ISSNe: $1549-2230$

http://dx.doi.org/10.5209/TEKN.74401

\title{
Riesgos de discriminación por discapacidad en la digitalización de la asistencia sanitaria
}

\author{
Mónica Otaola Barranquero'; Agustín Huete García ${ }^{2}$
}

Recibido: 22 de febrero de 2021 / Aceptado: 12 de abril de 2021 Open peer reviews

Resumen. Existen dos focos de atención relevantes que pueden generar espacios de desigualdad y discriminación en la población con discapacidad en las tecnologías de atención a la salud: i) aquellas relacionadas con necesidades específicas de asistencia, y ii) aquellas relacionadas con la accesibilidad de los dispositivos. Los artículos científicos recientes que tratan la telemedicina, $e$-health o el $m$-health, prestan escasa atención a la población con discapacidad, esto denota la necesidad de investigaciones y, en general, producción científica en este ámbito. La población con discapacidad debería ser objetivo prioritario en los planes de aplicaciones tecnológicas para la atención a la salud, no sólo en términos demográficos, sino en función de la especificidad de la demanda de atención sanitaria que se realiza en la actualidad, y porque la respuesta del sistema, tal y como está diseñado, resulta poco comunitaria y demasiado hospitalaria, esto es, más reactiva que preventiva. En caso contrario, si los desarrollos de los sistemas de telemedicina heredan esta concepción 'capacitista' de los sistemas sanitarios actuales, corren el riesgo de replicar los mismos defectos.

Palabras clave: accesibilidad; atención sanitaria; capacitismo; discriminación; telemedicina.

\section{[en] Risk of disability discrimination due to the digitalization of health care}

\begin{abstract}
There are two relevant focuses of attention that can generate spaces of inequality and discrimination in the population with disabilities in the area of health care technologies: i) those related to specific support needs, and ii) those related to the accessibility of devices. Recent scientific articles dealing with telemedicine, e-health or m-health, pay little attention to the population with disabilities, which indicates the need for research and, in general, scientific production in this field. People with disabilities should be a priority objective in plans for technological applications for health care, not only in demographic terms, but also based on the specificity of the present demand for health care and because the response of the system, as designed, is overly focused on acute hospital care to the exclusion of community-based and preventative care. If the developments of telemedicine systems inherit the ableist conception of current health systems, they run the risk of replicating their weaknesses. Keywords: ableism; accesibility; discrimination; health care; telemedicine.
\end{abstract}

Sumario. 1. Introducción. 2. La discapacidad como reto social y sanitario. 3. La salud como derecho para las personas con discapacidad. 4. Tendencias actuales en telemedicina y e-health. 5. Peculiaridades de atención sanitaria en la población con discapacidad. 6. Telemedicina y discapacidad: tendencias y retos. 7. Conclusión: dos zonas de riesgo de desigualdad por discapacidad en el desarrollo tecnológico en salud. 8. Referencias.

Cómo citar: Otaola Barranquero, M.; Huete García, A. (2021). Riesgos de discriminación por discapacidad en la digitalización de la asistencia sanitaria. Teknokultura. Revista de Cultura Digital y Movimientos Sociales, 18(2), 115-125. http:// dx.doi.org/10.5209/TEKN.74401

\section{Introducción}

Antes de la incorporación masiva de la tecnología telemática a la atención sanitaria, los estudios sobre desigualdades en salud ya habían determinado elementos discriminatorios de la población con discapacidad, principalmente en relación con la desatención de necesidades específicas por falta de recursos, por inaccesibilidad de espacios y dispositivos de atención, así como una alta frecuencia de rechazo o desconocimiento de las necesidades específicas por parte del personal sanitario (Organización Mundial de la Salud, 2020).

Aunque ciertamente la discapacidad es una cuestión anclada en una condición de salud, no siempre, aunque sí frecuentemente, las personas con discapacidad presentan necesidades específicas de asistencia sanitaria

\footnotetext{
Universidad de Salamanca (España)

E-mail: monicaotaola@usal.es; https://orcid.org/0000-0001-7277-8733

2 Universidad de Salamanca (España)

E-mail: ahueteg@usal.es; https://orcid.org/0000-0001-8652-5104 
(Jiménez y Huete, 2020) Por otra parte, las necesidades relacionadas con el diseño universal de los dispositivos tecnológicos son también muy variables, y pueden tener que ver con accesibilidad visual, auditiva, motórica y/o cognitiva. Existen, por tanto, dos focos de necesidades relevantes cuya desatención puede generar espacios de desigualdad y discriminación de la población con discapacidad en las tecnologías de atención a la salud: i) aquellas relacionadas con necesidades específicas de asistencia, y ii) aquellas relacionadas con la accesibilidad de los dispositivos tecnológicos actualmente en desarrollo.

En este artículo pretendemos proveer unas bases concretas para la reflexión prospectiva sobre focos de dificultad que el despliegue masivo de la telemedicina puede suponer para las personas con discapacidad, si no se toman las medidas adecuadas. Para ello, se propone inicialmente un marco de análisis fundamentado en el modelo social de la discapacidad, y su correlato legal fundamental, la Convención de Derechos de las Personas con Discapacidad (ONU, 2006). A partir de este marco se analizan las peculiaridades de la salud de las personas con discapacidad y la atención sanitaria que en la actualidad reciben, y que deberían ser tenidas en cuenta en los actuales desarrollos tecnológicos. Como consecuencia, se ofrece una visión prospectiva sobre los riesgos que los desarrollos tecnológicos pueden suponer si en su diseño no se contemplan las necesidades de la población con discapacidad.

Para la elaboración del texto se ha utilizado una metodología combinada. En primer lugar, se ha realizado una revisión de la literatura científica actual que aborda la cuestión de la discapacidad y la incorporación de la tecnología en la atención sanitaria. La revisión bibliográfica incluyó los descriptores $e$-health y telemedicine en los títulos, combinados con los términos trends y disability, disabled, ableism en los resúmenes y palabras clave. Esta combinación de términos y campos se ha aplicado para las bases de datos PsycInfo y Medline desde el año 2015, puesto que el objetivo de este artículo es ofrecer información actualizada relacionada con desarrollos tecnológicos en salud recientes. En total se han recuperado y revisado más de 150 artículos. Además, se ha realizado una explotación específica de la Encuesta Nacional de Salud (INE, 2017), buscando indicadores indirectos que puedan proveer información clave sobre las peculiaridades de salud y atención sanitaria de la población con discapacidad, que deberían ser tenidas en cuenta en los desarrollos en los desarrollos de telemedicina y e-health.

\section{La discapacidad como reto social y sanitario}

La discapacidad y las personas con discapacidad han recibido diferentes tratos, a lo largo del tiempo, por parte de la sociedad y de las instituciones políticas, que han sido compilados por la comunidad científica en diferentes modelos históricos de la discapacidad. Sin embargo, aunque, normalmente, se presenten de forma lineal y con una lógica cronológica, la realidad evidencia la coe- xistencia, en el presente, de varios de estos modelos, con mayor o menor intensidad (Evans, et al., 2017; Gabriel y Serrato, 2014).

El más arcaico es el modelo de 'prescindencia', tradicional o moral que está caracterizado «por una justificación religiosa de la discapacidad» (Palacios, 2008, p. 37) y por la creencia de que la discapacidad es un castigo de Dios (Evans et al., 2017). A nivel social se considera que la persona con discapacidad no tiene nada que aportar a la comunidad y por esto, se puede prescindir de la vida de las personas con discapacidad (Palacios, 2008). Se podría decir que este modelo de prescindencia persiste hoy en día en contextos cotidianos en los que las relaciones con personas con discapacidad son dominadas por el miedo, o el rechazo.

Por su parte, en el modelo 'médico' o rehabilitador la discapacidad se justifica en presupuestos clínicos, biólogos o científicos, y se identifica con la enfermedad (Evans et al., 2017; Jiménez y Huete, 2010; Palacios, 2008). La discapacidad se sitúa en el cuerpo, los problemas personales están causados por la discapacidad de la persona y las personas con discapacidad son víctimas que tienen que aprender a encargarse de las circunstancias que enfrentan (Evans et al., 2017) y que deben ser objeto de tratamiento y rehabilitación para obtener, si es posible, un funcionamiento normal, entendiendo 'normal' como 'sin discapacidad'. Sin entrar en detalles que no son objeto de este artículo, multitud de políticas públicas hoy en día permanecen dominadas por un concepto médico-rehabilitador (Huete, 2016), asimilacionista si se quiere, en el que el futuro de las personas con discapacidad es mejor si, mediante prótesis o tratamientos, consiguen minorar o incluso hacer desaparecer sus diferencias funcionales, también llamadas 'deficiencias'.

En las últimas décadas, ha cobrado protagonismo el denominado 'modelo social', en el que se entiende la discapacidad como el resultado de una organización social que discrimina a determinadas personas por su configuración corporal o funcional. Desde esta posición, se considera que las personas con discapacidad «tienen mucho que aportar a la sociedad o que, al menos, la contribución será en la misma medida que el resto de las personas (...) y está íntimamente relacionada con la inclusión y la aceptación de la diferencia») (Palacios, 2008, p. 104).

El modelo social de la discapacidad ha dado lugar a nuevos desarrollos conceptuales que buscan explorar la construcción social de la discapacidad, al estilo como se ha explicado la construcción social racista o machista. Esto ha llevado al perfeccionamiento, entre otros, del concepto de 'capacitismo' (ableism en inglés) que describe una construcción social en el que la ausencia de discapacidad es el «estado natural del ser humano y considera, además, este estado como altamente deseable» (McRuer, 2016, citado por Toboso, 2017, p. 4). Siguiendo a Campbell (2001):

el capacitismo se refiere a la red de creencias, procesos $\mathrm{y}$ prácticas que producen una forma particular de ser y del cuerpo, proyectado como perfecto, especie típica y por lo tanto esencial y plenamente humano. La disca- 
pacidad se presenta entonces como un estado disminuido del ser humano (Campbell, 2001, p. 44).

Un logro muy destacable de este modelo social de la discapacidad es la Convención Internacional sobre los Derechos de las personas con discapacidad (en adelante, Convención) de la Organización de las Naciones Unidas (ONU). La aprobación y posterior ratificación de esta Convención contempla la discapacidad como una cuestión de Derechos Humanos que debe ser atendida por los Estados que se atengan a la Convención, como es el caso de España. La Convención define la discapacidad como «una realidad que evoluciona y resulta de la interacción entre las personas con deficiencias y las barreras debidas a las actitudes y al entorno que evitan su participación plena y efectiva en la sociedad, en igualdad de condiciones con las demás» (ONU, 2006, preámbulo).

La Convención establece la accesibilidad entre sus principios generales (artículo 3), y en el artículo 9 que expresa:

...con el fin de que las personas con discapacidad puedan vivir en forma independiente y participar plenamente en todos los aspectos de la vida, los Estados Parte adoptarán medidas pertinentes para asegurar el acceso de las personas con discapacidad, en igualdad de condiciones con las demás, al entorno físico, el transporte, la información y las comunicaciones, incluidos los sistemas y tecnologías de la información (ONU, 2006).

La interpretación de la accesibilidad debe hacerse a la luz del concepto de barrera que señala el preámbulo de la Convención y en relación con el goce de todos los derechos plasmados en la misma. En cualquier caso, supone que la accesibilidad tiene dos sentidos, uno amplio y otro restringido: el amplio referido a los grandes derechos y en sentido restringido a productos, objetos, instrumentos, herramientas, entornos y servicios (de Asís, 2019).

De igual forma, la Convención define en su artículo 2 el concepto de 'diseño universal', reforzando la idea de que la accesibilidad no es sólo una cuestión propia de los contextos en los que las personas con discapacidad se desenvuelven:

Por 'diseño universal' se entenderá el diseño de productos, entornos, programas y servicios que puedan utilizar todas las personas, en la mayor medida posible, sin necesidad de adaptación ni diseño especializado. El 'diseño universal' no excluirá las ayudas técnicas para grupos particulares de personas con discapacidad, cuando se necesiten (ONU, 2006).

Según indica el Real Decreto Legislativo 1/2013 Ley General de los derechos de las personas con discapacidad en su artículo 2, el diseño universal, o diseño para todas las personas, es la actividad por la que se conciben o proyectan desde el origen, y siempre que sea posible, entornos, procesos, bienes, productos, servicios, objetos, instrumentos, programas, dispositivos o herramientas de tal forma que puedan ser utilizados por todas las personas, en la mayor extensión posible, sin necesidad de adaptación ni diseño especializado (Ley General de los derechos de las personas con discapacidad, artículo 2.1).

Las soluciones a los problemas de accesibilidad derivados de las dificultades de utilización del entorno construido precisan, la implementación del diseño universal para todas las personas en los bienes, servicios, productos o dispositivos por construir, y que son necesarios para la atención a la salud, de forma que, preventivamente, se eviten desarrollos tecnológicos que resulten inaccesibles para personas con discapacidad.

Por su parte, el Comité sobre los Derechos de las personas con discapacidad reforzó toda esta visión y, en su observación número 2 , reconoció que

la salud y la protección social seguirán estando fuera del alcance de las personas con discapacidad si no se tiene en cuenta un diseño universal y específicamente expresan que «toda la información y comunicación relativa a la prestación de la atención de salud debe estar disponible en lengua de señas, Braille, formatos electrónicos accesibles, escritura alternativa y modos, medios y formatos de comunicación... (CDPD ONU, 2014, p. 13).

\section{La salud como derecho para las personas con discapacidad}

Aunque legislativamente el acceso a los servicios de salud tiene una base bastante amplia, uno de los problemas a los que se enfrentan los sistemas de salud, desde la perspectiva del modelo social de la discapacidad, es la resolución de forma equilibrada de:

la integración de los modelos médicos y social sin que la adecuada consideración de las deficiencias derive de la estigmatización para el individuo y sin que el énfasis sobre las desventajas sociales impida prestar la necesaria atención a las necesidades en materia de prevención y rehabilitación de las discapacidades (Jiménez y Huete, 2010, p. 138).

En su articulado, la Convención reconoce que las personas con discapacidad tienen derecho a gozar del más alto nivel posible de salud sin discriminación por motivos de discapacidad, y obliga a los Estados a adoptar las medidas pertinentes para asegurar el acceso de las personas con discapacidad a servicios de salud incluida la rehabilitación relacionada con la salud (artículo 25) (ONU, 2006). En concreto a:

- Proporcionar a las personas con discapacidad programas y atención a la salud gratuitos.

- Proporcionar servicios de salud que necesiten las personas con discapacidad (...) incluidos los servicios destinados a prevenir la aparición de nuevas discapacidades.

- Proporcionar los servicios de salud lo más cerca posible de las comunidades de las personas con discapacidad, incluidas las zonas rurales.

- Exigir a los profesionales de la salud que presten a las personas con discapacidad atención de la 
misma calidad que a las demás personas sobre la base del consentimiento libre e informado.

Impedir que se nieguen, de manera discriminatoria, servicios de salud o de atención a la salud por motivos de discapacidad.

El acceso a los servicios de salud es un indicador principal para medir los resultados del sistema de bienestar social. En lo relativo al acceso a los servicios de salud se debe prestar atención a: i) el acceso físico a los hospitales y otras instalaciones sanitarias; ii) el equipamiento utilizado durante la prestación de la asistencia sanitaria; y iii) las herramientas de comunicación y educación de los pacientes (Annaswamy et al., 2020).

Desde un punto de vista capacitista, se enfatiza en el alcance de las representaciones simbólicas (Tamayo, Besoaín y Rebolledo, 2018) que tienen como consecuencia barreras institucionales a las que se somete a las personas con discapacidad (Bogart y Dunn, 2019). Esto es especialmente importante en, las denominadas, 'discapacidades invisibles' (Moral Cabrero, Huete García y Díez Villoria, 2020) como, por ejemplo, la discapacidad intelectual, las situaciones de salud crónicas, las enfermedades raras, aquellas que no han recibido un diagnóstico y los problemas de salud mental (Bogart y Dunn, 2019). A partir del estudio de Pachakins et al. (2018), Bogart y Dunn (2019) determinan que este estigma asociado a algunas discapacidades, especialmente las asociadas a enfermedades crónicas, tienen una repercusión en el estado de salud de los individuos con estas discapacidades.

El capacitismo, según Branco, Ramos y Hewstone (2019), es el -ismo (racismo, sexismo...) más perjudicial para la salud y el bienestar. Entre otras cosas, se encuentran prácticas en las políticas sanitarias actuales como, por ejemplo, la denegación de trasplantes, basadas en la creencia de que la vida de las personas con discapacidad vale menos que la vida de una persona sin discapacidad (NarioRedmond, Kemerling, y Silverman, 2019). A esto se añade el hecho de que muchas personas con discapacidad niegan u ocultan su discapacidad (Dunn, 2019).

En el plano de la sociología de la salud, desde hace varias décadas, se ha desarrollado el concepto de desigualdad(es) sociales en salud. Siguiendo a Whitehead y Dahlgreen (1991) uno de los enfoques de estas desigualdades sociales en salud implica asegurar que "cualquier política sanitaria preste atención a como el estado de salud, los riegos en salud y los servicios sanitarios están distribuidos en la población" (Whitehead y Dahlgreen, 1991, p. 1061). Esta conceptualización se ha ampliado con la definición de los determinantes sociales de la salud que se vinculan a las políticas públicas (Tamayo et al., 2018). La Organización Mundial de la Salud los define como: «las circunstancias en que las personas nacen, crecen, viven, trabajan y envejecen y (...) y son resultado de las políticas adoptadas» (Organización Mundial de la Salud, 2009).

En el caso de la discapacidad se trata como sinónimo y consecuencia del estado de salud, pero en muy pocas ocasiones se entiende en la gama de las condiciones sociales de la salud (Tamayo et al., 2018). Un nuevo en- foque implicaría reconocer la discapacidad como origen de las desigualdades en salud y no como resultado que, a su vez, unido al modelo social de la discapacidad, configuraría un marco apropiado para el diseño de nuevas políticas de salud pública (Tamayo et al., 2018).

Entre otros determinantes sociales de la salud se encuentra el acceso a la información y la tecnología. Sin embargo, este condicionante de salud no solo está relacionado con el acceso, sino con cómo la tecnología puede influir sobre otros determinantes generales de la salud como, por ejemplo, las relaciones sociales, los procesos culturales, los aspectos relacionados con el bienestar físico y mental, el acceso a la comunidad y la ampliación de información para tomar decisiones (Castiel y SanzValero, 2010).

\section{Tendencias actuales de la telemedicina y e-health}

Los términos telemedicina y e-health se usan indistintamente (Meier, Fitzgerald, y Smith, 2013), aunque existen diferencias que los tesauros bibliográficos no siempre las recogen; en concreto, la telemedicina tiene por objetivo reemplazar total o parcialmente las consultas presenciales y las visitas de enfermería, ahorrar costes y mejorar el cuidado en salud en aquellos lugares donde la calidad de los servicios de salud no es buena (Meier et al., 2013). Por su parte, la e-health utiliza la tecnología para proporcionar cuidados más allá de la clínica, como la salud pública y la teleeducación. $\mathrm{El}$ rápido crecimiento del big-data ha acelerado el desarrollo de $e$-health, en lo relativo al diagnóstico, tratamiento, cuidado de enfermería y toma de decisiones (Gu, et al., 2019).

Una de las tendencias actuales en telemedicina y $e$ health tiene que ver con las funcionalidades de la telefonía móvil en el manejo de los servicios de salud y la combinación del cuidado médico en el smart-health que se refiere al concepto novedoso asociado con las aplicaciones de nueva generación y de información (inteligencia artificial y los análisis big-data) (Gu et al., 2019). El uso del ambiente big-data, la interconexión de los datos a través de las comunidades y las plataformas de cuidados de salud, pueden ayudar a resolver el crecimiento en la población global y la mejora de las necesidades de los servicios de cuidados a personas mayores ( $\mathrm{Gu}$ et al., 2019).

El $m$-health se está utilizando para la creación de aplicaciones móviles para pacientes oncológicos (Kessel, Vogel, Schmidt-Graf, y Combs, 2016). Marzorati, et al. (2018) realizaron una revisión bibliográfica en la que identificaron diferentes formas de apoyar a cuidadores de personas con enfermedades oncológicas con tecnologías; en concreto: sesiones telefónicas y sistemas $e$-health en el que se favorecía el contacto con profesionales con diferentes propósitos relacionados con el bienestar (Marzorati et al., 2018).

En Israel, se ha puesto en marcha, con telemedicina, un historial en el que se graba un electrocardiograma inicial, transmitido por los propios pacientes simultáneamente durante su primera llamada al médico para 
que el centro de llamadas realice un análisis profesional instantáneo basándose en protocolos médicos que se actualizan periódicamente (Maor et al., 2020).

La gamificación, además de ser usada en entornos como el educativo, también se está usando en la $e$-health (Sardi, Idri y Fernández-Alemán, 2017). Los mecanismos del juego que se están aplicando a la e-health son los relacionados con las respuestas inmediatas, el uso de puntos y la integración del sistema social a través de mensajería instantánea (Sardi et al., 2017).

En Estados Unidos se están aplicando las consultas interactivas de vídeo, la transmisión de datos y consultas electrónicas, así como el intercambio con el registro médico electrónico (Baig, Antonescu-Turcu y Ratarasarn, 2016). También en este contexto, a principios de 2019 , se añadió una breve comunicación basada en chequeos virtuales; la evaluación remota de la información pregrabada del paciente; la consulta interprofesional por Internet; el registro virtual breve de audio para conocer si realmente se necesitaba una evaluación en persona, entre otros (Bajowala et al., 2020).

Como se ha mencionado con anterioridad la $e$-health puede ser empleada para la teleeducación de los pacientes. En concreto, en Canadá, se desarrolló un programa interactivo de un día en el que se enseñaba a las personas con artritis reumatoide a autogestionar su enfermedad de forma más efectiva (Kennedy et al., 2016). Este programa se diseñó a través de videoconferencias a las que también se unieron pacientes de las zonas rurales (Kennedy et al., 2016).

Las visitas médicas a través de la telemedicina también se han destinado a pacientes pediátricos (Ray, et al., 2019). Otro de los usos de la e-health para pacientes pediátricos es la monitorización remota de la diabetes y en los implantes cardiovasculares y la telepresencia robótica con cámaras que puedan hacer zoom (Sasangohar, et al., 2018).

La telemedicina también se está aprovechando para los triajes médicos de emergencia para personas mayores viviendo de forma independiente (Carolan, Grabowski, Mehrotra, y Hatfield, 2020). Una vez que se produce la llamada a los servicios de emergencia, y siempre que no sea una cuestión de vida o muerte, el servicio de atención tiene la posibilidad de ofrecer una video-consulta a través de una Tablet (Carolan et al., 2020). De la misma forma, se dedica la telemedicina para aquellos lugares rurales en los que no existen médicos especialistas en medicina de emergencia (Heppner et al., 2021).

En Brasil, la telemedicina también se aplica entre los diferentes trabajadores sanitarios y, en concreto, para poner en contacto a algunos médicos con especialistas en cardiología cuando se trataba de pacientes con enfermedades del corazón (Macedo et al., 2016).

La aplicación de la Inteligencia Artificial está cada vez más extendida en la comunidad médica y una de las ramas en las que, en la actualidad, se aplica es la oftalmología (Jheng et al., 2020). En concreto, se usan los algoritmos de la Inteligencia Artificial para detectar algunas enfermedades sobre las que se presentan algunas cuestiones éticas como puede ser el buen testeo de estos algoritmos antes de ser usados (Jheng et al., 2020). Se destacan como posibles vías de implementación la adopción de tratamientos individualizados basados en cada paciente y desarrollados en los dispositivos móviles de cada paciente y enviados a su vez, a los médicos para su examen (Jheng et al., 2020).

Se han creado otros sistemas de e-health, basados en la inteligencia artificial, para el tratamiento de personas con adicciones apoyados en tecnologías como: la creación de mundos virtuales, aplicaciones de teléfono, videoconferencias (Molfenter et al., 2015).

En el contexto estadounidense, las barreras que se detectan, en general, para la implementación de la telemedicina son: las reticencias de los pacientes para recibir consultas virtuales; por parte de la comunidad médica se detecta la necesidad de tiempo para aprender las nuevas metodologías y las reticencias por creer que rompen sus metodologías de trabajo presencial con los pacientes; la dificultad para recoger los consentimientos informados y los retos para cubrir legalmente la mala praxis (Dooley, Houssaye y Baum, 2020).

Sin embargo, en países como Estados Unidos, la pandemia de la covid-19 ha supuesto la generalización de algunas medidas de telemedicina que se volvieron oficiales, mientras que antes se hacían de forma voluntaria por parte del personal sanitario (Bajowala et al., 2020).

\section{Peculiaridades de salud y atención sanitaria en la población con discapacidad}

El número de personas con discapacidad está aumentando debido en parte al envejecimiento de la población y al incremento de la prevalencia de enfermedades crónicas. Según estimaciones de la Organización Mundial de la Salud (2011) más de 1.000 millones de personas viven con algún tipo de discapacidad. En España, según las fuentes que utilicemos para la estimación de la población, viven aproximadamente 4 millones de personas con discapacidad (IMSERSO, 2019; INE, 2008). Según datos de Encuesta de Integración Social y Salud (INE, 2012), ya hace casi una década el $28,2 \%$ de las personas con discapacidad en España encontraba barreras para usar Internet, cuestión mucho más relevante en el contexto actual de desarrollos tecnológicos en atención sanitaria.

La Encuesta Nacional de Salud (INE, 2017) permite obtener información sobre la salud y asistencia sanitaria a personas con discapacidad, distinguiendo algunas tipologías importantes: discapacidades de la visión, audición físicas y mentales. Estas últimas, sin embargo, no permiten distinguir entre población con problemas de salud mental y personas con discapacidad intelectual.

Un primer indicador que explica las diferencias entre población con y sin discapacidad es el estado de salud percibido. Tres cuartas partes de la población sin discapacidad reconoce un estado de salud bueno o muy bueno, mientras que en la población general con discapacidad esta percepción positiva apenas asciende al $20 \%$, siendo incluso inferior en población con discapacidad de origen físico o mental (Gráfico 1). 
Gráfico 1. Estado de salud percibido en los últimos 12 meses.

Fuente: elaboración propia a partir de INE (2017)

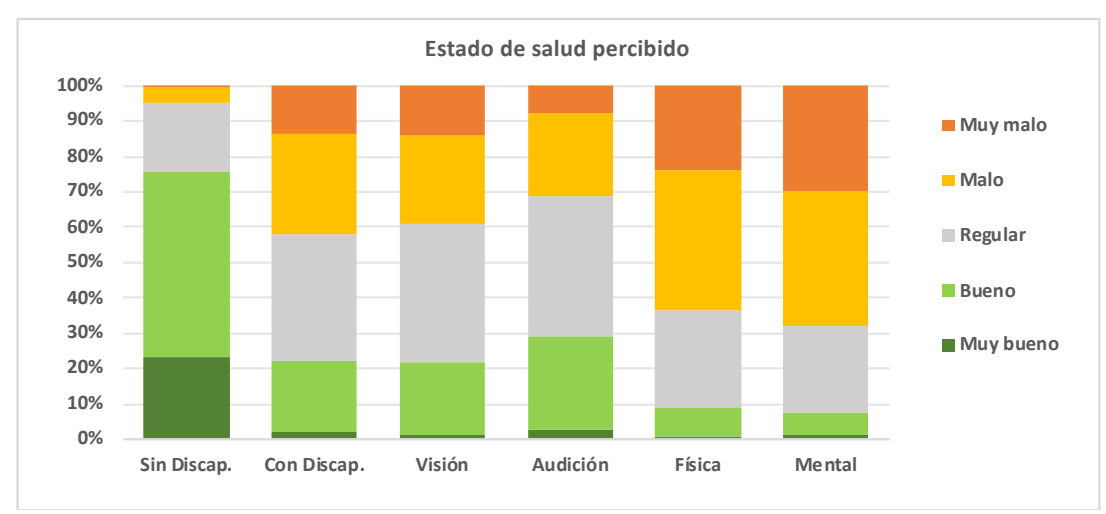

La presión que ejerce la población con discapacidad sobre el sistema sanitario es a su vez netamente superior y heterogénea, en todos los niveles del sistema. Las demandas de atención de la población sin discapacidad se adaptan perfectamente al escalamiento del sistema, siendo así más alta sobre la atención primaria, después sobre la de especialidades e inferior en la atención hospitalaria
(Grafico 2). Este indicador denota claramente que el sistema sanitario está diseñado para responder a las demandas de atención de una población sin discapacidad, pero no así para la población con discapacidad, que no sólo requiere atención con más frecuencia, sino en muchos casos directamente hospitalaria, esto es, no atendida por los recursos comunitarios (atención primaria).

Gráfico 2. Consultas anuales a atención primaria, especialistas y hospitalaria. Fuente: elaboración propia a partir de INE (2017)

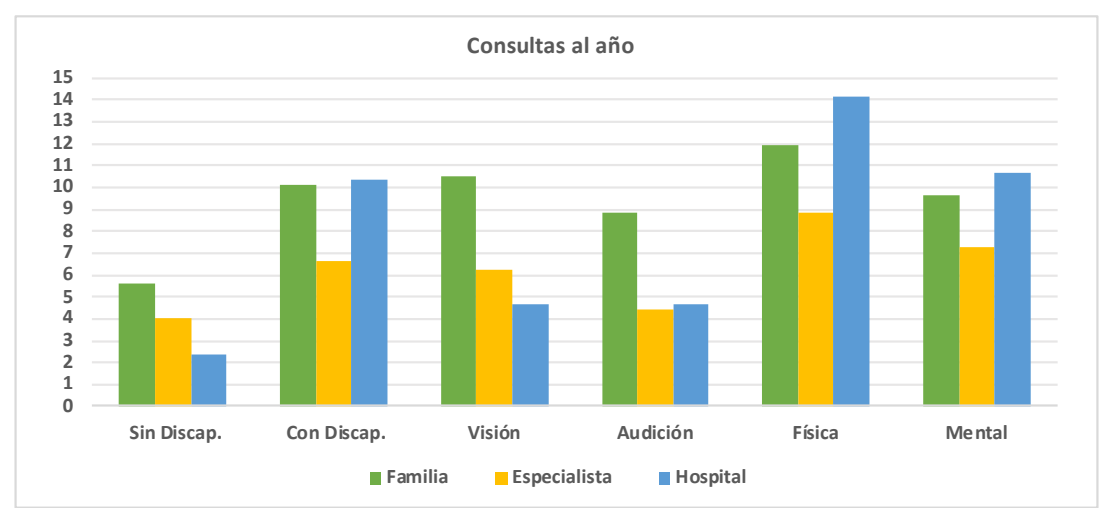

La Encuesta Nacional de Salud (INE, 2017), no contiene información específica sobre uso de sistemas de telemedicina, pero sí permite conocer las posibilidades de uso del teléfono en la población encuestada, siendo esta posible sin dificultad para la práctica totalidad de la población sin discapacidad, pero no así para la población con discapacidad, especialmente de la visión, física y mental.

Gráfico 3. Dificultad para utilizar el teléfono.

Fuente: elaboración propia a partir de INE (2017)

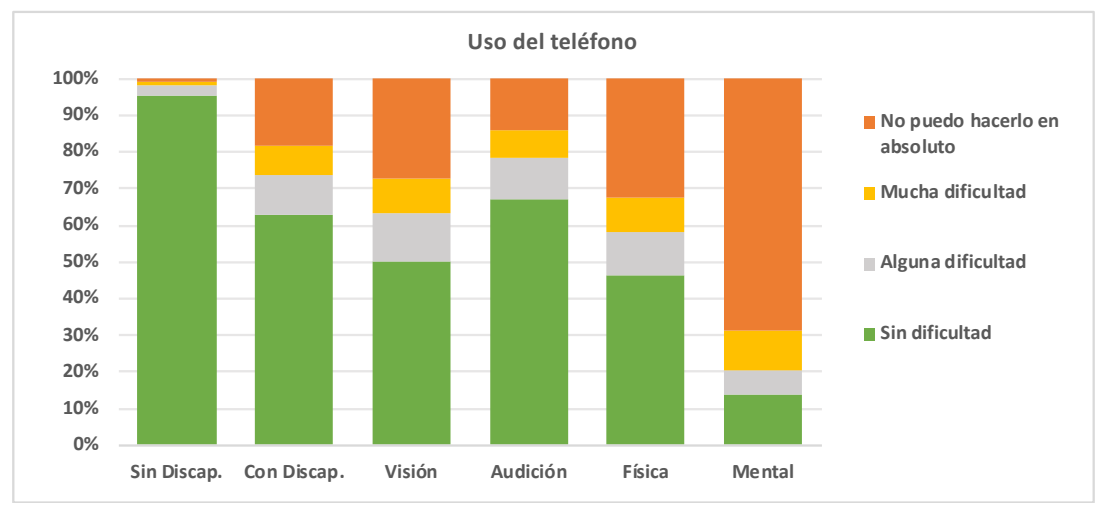


Finalmente, se puede obtener información indirecta sobre las necesidades de la población atendiendo a las demandas de consultas domiciliarias. Según la Encuesta Nacional de Salud (INE, 2017), 164.575 personas en España solicitaron consultas domiciliarias en los 12 meses anteriores a la encuesta. De ellas, 22.693, esto es el 14\% eran personas sin discapacidad (Gráfico 3), lo que nos da una idea de que una amplia mayoría de quienes precisan atención médica en el domicilio, son personas con discapacidad (Gráfico 4).

Gráfico 4. Atención domiciliaria por una enfermera o matrona.

Fuente: elaboración propia a partir de INE (2017)

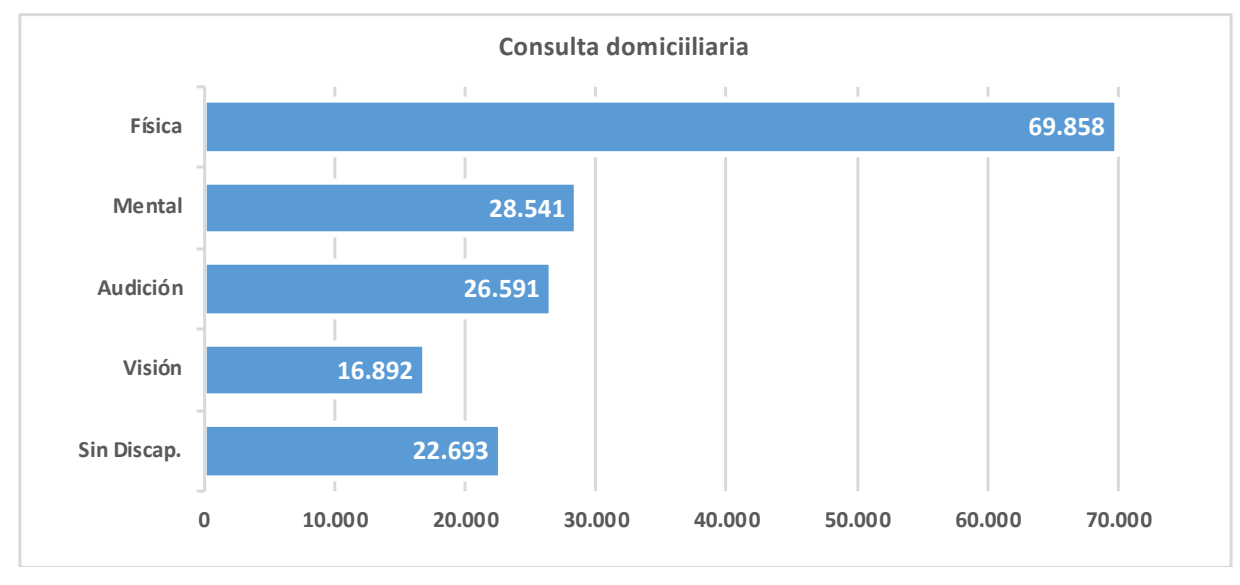

\section{Telemedicina y discapacidad: tendencias y retos}

Los artículos científicos recientes que tratan la telemedicina, e-health o el m-health, lo hacen sin ninguna distinción sobre lo que son las enfermedades y lo que es la discapacidad, es decir, no existe un tratamiento preciso de la discapacidad. En la revisión bibliográfica realizada han sido muy escasas las referencias asociadas a telemedicina y discapacidad, lo cual denota necesidad de producción científica en este ámbito.

Una de las aplicaciones de la telemedicina o de la $e$ health es la tele-rehabilitación en la que se utilizan televisitas, llamadas telefónicas y realidades virtuales (Altilio et al., 2015). La ventaja de la tele-rehabilitación es poder seguir con la rehabilitación aún en casa y fuera de las instalaciones hospitalarias. Sin embargo, todavía no son suficientes las evidencias para establecer la posibilidad de realizar diagnósticos y poder disponer de un examen estandarizado para cualquier tratamiento y hacer un seguimiento de los resultados de la tele-rehabilitación (Altilio et al., 2015).

La telemedicina también se está utilizando para la población con problemas de salud mental y la denominación que recibe es e-mental health (Beentjes et al., 2018). Aunque su uso está cada vez más extendido, su efectividad no está comprobada (Beentjes et al., 2018). Esta técnica de telemedicina se puede usar para aquellas personas con enfermedades mentales residentes en el medio rural (Creedon et al., 2020).

En los Países Bajos utilizaron una técnica mixta, denominada 'e-IMR', para la recuperación de los pacientes con enfermedades mentales (Bajowala et al., 2020). Esta técnica se desarrollaba en una plataforma con videos ilustrativos, con testimonios de otros participantes para motivar el proceso de recuperación, formularios de resolución de problemas y de registro y una página de monitoreo de los síntomas (Bajowala et al., 2020).
La educación saludable es otro de los usos de la $e$ health que se han implementado para jóvenes con movilidad reducida. Este programa incluía ejercicios y planes de comidas saludables a través de dispositivos móviles y reuniones presenciales con los educadores (Berglind et al., 2018). El mismo tipo de programa se aplicó a la población con discapacidad intelectual (Neumeier et al., 2017). La telemedicina también se ha usado para niños con discapacidad intelectual en los colegios rurales, reduciéndose así los costes de los viajes (Langkamp, McManus y Blakemore, 2015).

Los temas para los que se aplican la telemedicina en los adultos con discapacidad intelectual son: tecnología asistida; páginas web para apoyar las actividades básicas de la vida diaria; videollamadas; tele-psiquiatría, realidad virtual; control del peso; salud bucal; tele-cuidados para la vida independiente y los registros médicos electrónicos (Vázquez et al., 2018).

Ahora bien, las barreras de acceso a los cuidados de salud se refieren a la tecnología, en concreto, al acceso a conexión de internet, especialmente en el mundo rural en el que viven muchas personas con discapacidad y en el que no existe la suficiente inversión para proveer un acceso adecuado (Annaswamy et al., 2020). A esto se une las barreras de los interfaces como pueden ser los lectores de pantallas, la 'lengua de signos' y los contrastes; además de las comunicativas para personas con discapacidad intelectual o psicosociales (Annaswamy et al., 2020).

La aplicación de telemedicina de forma generalizada precisa atención a las barreras en la legislación en lo que se refiere a los estándares de accesibilidad y los plazos recogidos en estas legislaciones para implementarlos (Annaswamy et al., 2020). En Reino Unido, por ejemplo, se ha incluido en algunos planes de salud para las personas con discapacidad intelectual en el proceso de envejecimiento, que la telemedicina solo será un com- 
plemento a las consultas presenciales y en ningún caso sustituirá a estas (Madhavan, 2019).

La telemedicina aplicada a la población con discapacidad intelectual trae consigo una serie de cuestiones éticas a tener en cuenta como, por ejemplo, el consentimiento informado o la necesidad de asegurar el entendimiento de las preguntas que se hagan a los pacientes (Perry, Beyer y Holm, 2009), entre otras cuestiones relacionadas con la población con discapacidad intelectual y la telemedicina como son las relacionados con la ética y la bioética en el proceso salud-enfermedad-atención.

Más de un $90 \%$ de las personas con Trastorno del Espectro Autista muestra dificultades para la comunicación de su estado de salud (Merino et al., 2010). Estas barreras comunicativas en las personas con discapacidad intelectual pueden ser eliminadas con la utilización de pictogramas u otros métodos de adaptación, a las que se pueden acceder gracias al progreso de la tecnología. Bonanno (2016), desarrolló, para su Tesis Doctoral, una página web, basada en la telemedicina que tenía por objetivo aportar a los médicos una historia médica visual incorporada a la propia aplicación de registro e historias médicas (Bonanno, 2016).

En España, se ha desarrollado el Programa '¿Qué me pasa doctor? Derecho a mi salud' con el mismo objetivo, en el que se crea una tipología de apoyos visuales formada por pruebas médicas, diagnósticos y trámites; además de un comunicador interactivo, a través de la aplicación Mefacilyta (Guerra Rivas, 2019).

En algunos países se están desarrollando tecnologías para que no sea necesaria la presencia constante de personal sociosanitario en las instituciones; estas tecnologías son capaces de medir cuantas veces una persona ha acudido al baño durante la noche o cuando una persona lleva mucho tiempo sin moverse (Perry et al., 2009). Estas tecnologías parecen benevolentes, sin embargo, se pueden considerar como una intrusión en la privacidad y libertad de los usuarios. Con esto, a pesar de la falta de personal sociosanitario, las instituciones y los propios usuarios tienen que medir sus beneficios frente a las posibles desventajas en términos de monitorear su intimidad (Perry et al., 2009).

Otros investigadores (Dorsey, Okun y Bloem, 2020) afirman que la telemedicina tiene ventajas para las personas con discapacidad, entre otras: cuidados accesibles, conveniencia, mejora del confort, confianza para los pacientes y las pacientes y ahora, con la covid-19, menos riesgo de contagio (Dorsey et al., 2020). Además, la telemedicina hace que los médicos puedan evaluar a los pacientes en sus contextos naturales (Dorsey et al., 2020).

La pandemia mundial de la covid-19 ha marcado un antes y un después en las barreras a las que se enfrentan las personas con discapacidad. Además, esta pandemia ha acelerado muchos procesos, entre ellos, la adopción de la telemedicina en detrimento de las consultas tradicionales cara a cara, en las que se podían revertir las barreras de acceso (Annaswamy et al., 2020). Así, la telemedicina ha dejado de considerarse como un complemento a las consultas tradicionales (Annaswamy et al., 2020).

\section{Conclusión: dos zonas de riesgo de desigualdad por discapacidad en el desarrollo tecnológico en salud}

En este texto, hemos tratado de exponer dos focos de riesgos cuya desatención pueden generar espacios de desigualdad y discriminación de la población con discapacidad en las tecnologías de atención a la salud: i) aquellas relacionadas con necesidades específicas de asistencia, y ii) aquellas relacionadas con el diseño universal y la accesibilidad de los dispositivos tecnológicos en desarrollo.

Comenzando por el primero, la discapacidad, en la mayoría de las ocasiones y en función de una concepción médica generalizada sociopolíticamente, se usa como sinónimo y resultado del estado de salud. Sin embargo, nuevas perspectivas se han desarrollado sobre cómo implementar y diseñar políticas públicas dirigidas a la población con discapacidad. Para ello, la Convención impulsa a los Estados Parte por ella vinculados, a implementar medidas basadas en los Derechos Humanos, entre ellas, una atención sanitaria universal y para todas las personas.

Aunque la información sobre necesidades específicas de la población con discapacidad ante los sistemas de salud tradicionales es escasa, sabemos que se trata de una población que ejerce una presión específica sobre los recursos sanitarios, y que no recibe una respuesta adecuada, siendo generalmente reactiva y hospitalaria. Por tipos de discapacidad, esta discrepancia entre necesidades de la población y respuesta del sistema requiere una atención diferenciada.

Lo que sabemos sobre las necesidades actuales de atención sanitaria de la población con discapacidad necesariamente ha de ser tenido en cuenta a la hora de diseñar dispositivos tecnológicos de atención a la salud, desde la óptica del diseño universal, y se podría resumir en: i) el nivel de satisfacción de necesidades de salud desde el sistema sanitario es mucho más bajo en la población con discapacidad, ii) los dispositivos diseñados para la atención a la población general (sin discapacidad) resultan habitualmente insuficientes para la población con discapacidad, iii) los dispositivos basados en atención telemática deben ser diseñados bajo los estándares del diseño universal, y iv) cualquier solución tecnológica tendente a incrementar o mejorar la atención sanitaria domiciliaria tendrá como destinatario frecuente a personas con discapacidad.

El desarrollo de los planes de telemedicina en atención sanitaria precisa la aplicación de un diseño universal y para todas las personas que no replique hacia el futuro la concepción 'capaticista' actual de los sistemas de salud, construidos en función de una demanda en la que las necesidades de la población con discapacidad no han sido tenidas en cuenta, lo cual ha tenido consecuencias perjudiciales que se materializan en un peor estado de salud, y en una precaria atención sanitaria.

En relación con el segundo de los focos de atención de este artículo, el rápido desarrollo de la telemedicina, bajo los paraguas del $m$-health y de la e-health, supone una transformación integral de los dispositivos de atención sanitaria. Las tendencias de la telemedicina 
se dirigen no solo hacia la sustitución de las consultas presenciales, sino también a triajes, consultas entre diferentes especialidades, la aplicación de la Inteligencia Artificial y la gamificación. Al fuerte impulso propio del desarrollo tecnológico, se ha unido la necesidad de aplicación en tiempos de pandemia de covid-19, en la que el distanciamiento personal y la no presencialidad se han convertido en necesidad vital.

Los desarrollos actuales en telemedicina y e-health parecen replicar esta ausencia de un diseño universal y para todas las personas, cuestión que puede afectar a la atención sanitaria de las personas con discapacidad. Tal como se ha mostrado, los avances en este terreno en escasas ocasiones contemplan la discapacidad como una población objetivo para tener en cuenta, y proyectan el uso masivo de tecnologías que pueden resultar ineficaces, inaccesibles, y cuyo uso ya hoy en día, más allá de las aplicaciones en salud, resulta, de hecho, problemático.
La escasa atención académica hacia esta cuestión se ejemplifica en la poca distinción que se ofrece en la literatura científica entre discapacidad y enfermedad, dándose la necesidad de más investigación sobre las necesidades de las personas con discapacidad y las distinciones entre las barreras sociales hacia la discapacidad y las enfermedades específicas.

En definitiva, la población con discapacidad debería ser objetivo prioritario en los planes de atención a la salud, no sólo en términos demográficos, sino en función de la especificidad de la demanda de atención sanitaria que realiza en la actualidad, y para la que la respuesta del sistema, tal y como está diseñado, resulta poco comunitaria y demasiado hospitalaria, esto es, más reactiva que preventiva. En consecuencia, si la implementación y desarrollo de los sistemas de telemedicina heredan esta concepción 'capacitista' de los sistemas, y se construyen sobre ella, replicarán con alta probabilidad los mismos defectos.

\section{Referencias}

Adusumilli, G., Joseph, S. E., Samaan, M. A., Schultz, B., Popovic, T., Souza, R. B. y Majumdar, S. (2017). Iphone sensors in tracking outcome variables of the 30 -second chair stand test and stair climb test to evaluate disability: Cross-sectional pilot study. JMIR mHealth and uHealth, 5(10), 1-11. https://doi.org/10.2196/mhealth.8656

Altilio, R., Liparulo, L., Panella, M., Proietti, A. y Paoloni, M. (2015). Multimedia and Gaming Technologies for Telerehabilitation of Motor Disabilities [Leading Edge]. IEEE Technology and Society Magazine, 34(4), 23-30. https://doi.org/10.1109/ MTS.2015.2494279

Annaswamy, T. M., Verduzco-Gutierrez, M. y Frieden, L. (2020). Telemedicine barriers and challenges for persons with disabilities: COVID-19 and beyond. Disability and Health Journal, 13(4), 100973. https://doi.org/10.1016/j.dhjo.2020.100973

Baig, M. M., Antonescu-Turcu, A. y Ratarasarn, K. (2016). Impact of Sleep Telemedicine Protocol in Management of Sleep Apnea: A 5-Year VA Experience. Telemedicine and e-Health, 22(5), 458-462. https://doi.org/10.1089/tmj.2015.0047

Bajowala, S. S., Milosch, J. y Bansal, C. (2020). Telemedicine Pays: Billing and Coding Update. Current Allergy and Asthma Reports, 20(10). https://doi.org/10.1007/s11882-020-00956-y

Beentjes, T. A. A., Goossens, P. J. J., Vermeulen, H., Teerenstra, S., Nijhuis-Van Der Sanden, M. W. G. y Van Gaal, B. G. I. (2018). E-IMR: E-Health added to face-to-face delivery of Illness Management \& Recovery programme for people with severe mental illness, an exploratory clustered randomized controlled trial. BMC Health Services Research, 18(1), 1-10. https://doi. org/10.1186/s12913-018-3767-5

Berglind, D., Nyberg, G., Willmer, M., Persson, M., Wells, M. y Forsell, Y. (2018). An e-health program versus a standard care supervised health program and associated health outcomes in individuals with mobility disability: study protocol for a randomized controlled trial. Trials, 19(1), 1-9. https://doi.org/10.1186/s13063-018-2646-Z

Bogart, K. R. y Dunn, D. S. (2019). Ableism Special Issue Introduction. Journal of Social Issues, 75(3), 650-664. https://doi. org/10.1111/josi.12354

Bonanno, G. (2016). The importance of a pictorial medical history in assisting medical diagnosis of individuals with intellectual disabilities: A telemedicine approach. Dissertation Abstracts International: Section B: The Sciences and Engineering, 76(10$\mathrm{B}(\mathrm{E})$.

Branco, C., Ramos, M. R. y Hewstone, M. (2019). The Association of Group-Based Discrimination with Health and Well-Being: A Comparison of Ableism with Other 'Isms'. Journal of Social Issues, 75(3), 814-846. https://doi.org/10.1111/josi.12340

Campbell, F. A. K. (2001). Inciting Legal Fictions: Disability's Date with Ontology and the Ableist Body of the Law. Griffith Law Review, 10(1), 43-62.

Carolan, K., Grabowski, D. C., Mehrotra, A. y Hatfield, L. A. (2020). Use of telemedicine for emergency triage in an independent senior living community: Mixed methods study. Journal of Medical Internet Research, 22(12). https://doi.org/10.2196/23014

Castiel, L. D. y Sanz-Galero, J. (2010). El acceso a la información como determinante social de la salud. Nutrición Hospitalaria, 25(3), 26-30. https://bit.ly/3uKXYXv

Comité CDPD ONU. (2014). Observación general No 2 Art. 9: Accesibilidad (Vol. 03316).

Creedon, T. B., Schrader, K. E., O’Brien, P. L., Lin, J. R., Carroll, C. D. y Mulvaney-Day, N. (2020). Rural-nonrural differences in telemedicine use for mental and substance use disorders among medical beneficiaries. Psychiatric Services, 71(8), 756-764. https://doi.org/10.1176/appi.ps.201900444

de Asís, R. (2019). La Accesibilidad Universal desde la Perspectiva Jurídica. En EKARTUZ de Gipuzkoa (Ed.), Congreso Internacional Discapacidad, Derechos e Inclusión, Guipúzcoa, España.

Dooley, A. B., Houssaye, N. de la, y Baum, N. (2020). Use of Telemedicine for Sexual Medicine Patients. Sexual Medicine Reviews, 8(4), 507-517. https://doi.org/10.1016/j.sxmr.2020.06.001

Dorsey, E. R., Okun, M. S. y Bloem, B. R. (2020). Care, Convenience, Comfort, Confidentiality, and Contagion: The 5 C's that Will Shape the Future of Telemedicine. Journal of Parkinson's Disease, 10(3), 893-897. https://doi.org/10.3233/JPD-202109 
Dunn, D. S. (2019). Outsider Privileges Can Lead to Insider Disadvantages: Some Psychosocial Aspects of Ableism. Journal of Social Issues, 75(3), 665-682. https://doi.org/10.1111/josi.12331

Engel, G. L. (1992). The need for a new medical model: A challenge for biomedicine. Family Systems Medicine, 10(3), $317-331$. https://doi.org/10.1521/pdps.2012.40.3.377

Evans, N. J., Broido, E. M., Brown, K. R. y Wilke, A. K. (2017). Disability in Higher Education. En Jossey-Bass A willey Brand (Vol. 53).

Gabriel, J. y Serrato, M. de las M. (2014). Del padecimiento a la diversidad: un camino hermenéutico From complaint to diversity : a hermeneutic. Revista Española de Discapacidad, 2(2), 185-206. http://dx.doi.org/10.5569/2340-5104.02.02.10

Gu, D., Li, T., Wang, X., Yang, X. y Yu, Z. (2019). Visualizing the intellectual structure and evolution of electronic health and telemedicine research. International Journal of Medical Informatics, 130(August), 103947. https://doi.org/10.1016/j. ijmedinf.2019.08.007

Guerra Rivas, A. (2019). Uso de aplicaciones tecnológicas en la atención sanitaria de personas con Trastorno del Espectro Autista (TEA). Actas de Coordinación Sociosanitaria, 25.

Heppner, S., Mohr, N. M., Carrter, K. D., Ulrich, F., Merchant, K. A. S. y Ward, M. M. (2021). HRSA's evidence-based teleemergency network grant program : Multi-site prospective cohort analysis across six rural emergency department telemedicine networks. PLoS One, 16(1), 1-13. https://doi.org/10.1371/journal.pone.0243211

Huete García, A. (2016). El diagnóstico como recurso sanitario y social. Actas de Coordinación sociosanitaria, 17, 19-28. http:// www.newhealthfoundation.org/web/wp-content/uploads/2016/08/actas_julio.pdf

IMSERSO (2019). Base Estatal de datos de personas con discapacidad. Madrid. Recuperado de: https://bit.ly/3uHcZJU

INE (2008). Encuesta de discapacidad, autonomía personal y situaciones de dependencia. Recuperado de: www.ine.es

INE (2008). Encuesta de Integración Social y Salud. Recuperado de: www.ine.es

INE y Ministerio de Sanidad, Consumo y Bienestar Social (2017). Encuesta Nacional de Salud. Madrid. Recuperado de: https:// bit.ly/3dRBihh

Jheng, Y., Kao, C., Yarmishyn, A. A., Chou, Y. y Hsu, C. (2020). The era of artificial intelligence-based individualized telemedicine is coming. Journal of Chinese Medical Association, 83, 981-983. https://doi.org/10.1097/JCMA.0000000000000374

Jiménez Lara, A. y Huete García, A. (2010). Políticas públicas sobre discapacidad en España. Hacia una perspectiva basada en los derechos. Politica y Sociedad, 47(1), 137-152. https://revistas.ucm.es/index.php/POSO/article/view/POSO1010130137A

Jiménez Lara, A. y Huete García, A. (2020). Personas con discapacidad: análisis estadístico. Informe Olivenza 2019 sobre la situación de la discapacidad. Observatorio Estatal de la Discapacidad (OED). https://www.observatoriodeladiscapacidad. info/informe-olivenza-2019-sobre-la-situacion-general-de-la-discapacidad-en-espana/

Kennedy, C. A., Warmington, K., Flewelling, C., Shupak, R., Papachristos, A., Jones, C., ... Hogg-Johnson, S. (2016). A prospective comparison of telemedicine versus in-person delivery of an interprofessional education program for adults with inflammatory arthritis. Journal of Telemedicine and Telecare, 23(2), 197-206. https://doi.org/10.1177/1357633X16635342

Kessel, K. A., Vogel, M. M., Schmidt-Graf, F. y Combs, S. E. (2016). Mobile Apps in Oncology: A Survey on Health Care Professionals' Attitude Toward Telemedicine, mHealth, and Oncological Apps. Journal of medical Internet research, 18(11), e312. https://doi.org/10.2196/jmir.6399

Koceska, N., Koceski, S., Zobel, P. B., Trajkovik, V. y García, N. (2019). A telemedicine robot system for assisted and independent living. Sensors (Switzerland), 19(4), 1-17. https://doi.org/10.3390/s19040834

Lai, F. H. yin, Yan, E. W. hung, Yu, K. K. ying, Tsui, W. S., Chan, D. T. hoi, y Yee, B. K. (2020). The Protective Impact of Telemedicine on Persons with Dementia and Their Caregivers During the COVID-19 Pandemic. American Journal of Geriatric Psychiatry, 28(11), 1175-1184. https://doi.org/10.1016/j.jagp.2020.07.019

Langkamp, D. L., McManus, M. D. y Blakemore, S. D. (2015). Telemedicine for children with developmental disabilities: A more effective clinical process than office-based care. Telemedicine and e-Healthe-Health, 21(2), 110-114. https://doi.org/10.1089/ tmj.2013.0379

Macedo, T. A., De Barros E Silva, P. G. M., Simões, S. A., Okada, M. Y., Garcia, J. C. T., Sampaio, M. C., ... Furlan, V. (2016). Impact of Chest Pain Protocol with Access to Telemedicine on Implementation of Pharmacoinvasive Strategy in a Private Hospital Network. Telemedicine and e-Healthe-Health, 22(7), 549-552. https://doi.org/10.1089/tmj.2015.0178

Madhavan, G. (2019). Telepsychiatry in intellectual disability psychiatry: literature review. BJPsych Bulletin, 43(4), 167-173. https://doi.org/10.1192/bjb.2019.5

Maor, E., Abend, Y., Ganem, D., Kusniec, F., Grosman-Rimon, L., Elbaz-Greener, G., ... Amir, O. (2020). Sex Disparities in First Medical Contact of Patients with Suspected Acute Coronary Syndrome Using Telemedicine Technology. Telemedicine and e-Healthe-Health, 26(4), 411-418. https://doi.org/10.1089/tmj.2019.0036

Marzorati, C., Renzi, C., Russell-Edu, S. W. y Pravettoni, G. (2018). Telemedicine use among caregivers of cancer patients: Systematic review. Journal of Medical Internet Research, 20(6). https://doi.org/10.2196/jmir.9812

Meier, C. A., Fitzgerald, M. C. y Smith, J. M. (2013). EHealth: Extending, enhancing, and evolving health care. Annual Review of Biomedical Engineering, 15(April), 359-382. https://doi.org/10.1146/annurev-bioeng-071812-152350

Merino Marínez, M., García Alonso, M. A., Martínez Marín, M. A., Arnáiz Sancho J. S., de la Iglesia Gutiérrez M., Hortiguela Terrel, V., Nieto Maestre, M., García Fernández, C., Esteban Heras, N. (2010). El acceso a la presentación de asistencia sanitaria de las personas con TEA. Un problema sin resolver. Siglo Cero. Revista Española sobre Discapacidad Intelectual, 41(4), 77.

Molfenter, T., Boyle, M., Holloway, D. y Zwick, J. (2015). Trends in telemedicine use in addiction treatment. Addiction Science and Clinical Practice, 10(1), 1-9. https://doi.org/10.1186/s13722-015-0035-4

Moral Cabrero, E., Huete García, A. y Díez Villoria, E. (2020). ¿Soy lo que ves? Microagresiones capacitistas y visibilidad de la discapacidad. Revista Española de Discapacidad (REDIS), 8(2), 7-31. https://doi.org/10.5569/2340-5104.08.02.01

Morris, J., Jones, M., Thompson, N., Wallace, T. y Deruyter, F. (2019). Clinician perspectives on mrehab interventions and technologies for people with disabilities in the united states: A national survey. International Journal of Environmental Research and Public Health, 16(21), 1-11. https://doi.org/10.3390/ijerph16214220 
Nario-Redmond, M. R., Kemerling, A. A. y Silverman, A. (2019). Hostile, Benevolent, and Ambivalent Ableism: Contemporary Manifestations. Journal of Social Issues, 75(3), 726-756. https://doi.org/10.1111/josi.12337

Neumeier, W. H., Guerra, N., Thirumalai, M., Geer, B., Ervin, D. y Rimmer, J. H. (2017). POWERSforID: Personalized Online Weight and Exercise Response System for Individuals with Intellectual Disability: Study protocol for a randomized controlled trial. Trials, 18(1), 1-11. https://doi.org/10.1186/s13063-017-2239-2

ONU (2006). Convención sobre los Derechos de las personas con discapacidad. Recuperado de: https://www.un.org/esa/socdev/ enable/documents/tccconvs.pdf

Organización Mundial de la Salud (2009). Subsanar las desigualdades de una generación. Alvanzar la equidad sanitaria actuando sobre los determinantes sociales de la salud. Recuperado de: https://bit.ly/3my7ZV1

Organización Mundial de la Salud (2020). Discapacidad y salud. Recuperado de: https://www.who.int/es/news-room/fact-sheets/ detail/disability-and-health

Palacios, A. (2008). El modelo social de la discapacidad: Orígenes, caracterización y plasmación en la Convención Internacional sobre los Derechos de las Personas con Discapacidad. En CINCA. Recuperado de: https://bit.ly/3scQKda

Perry, J., Beyer, S. y Holm, S. (2009). Assistive technology, telecare and people with intellectual disabilities: Ethical considerations. Journal of Medical Ethics, 35(2), 81-86. https://doi.org/10.1136/jme.2008.024588

Ray, K. N., Shi, Z., Poon, S. J., Uscher-Pines, L. y Mehrotra, A. (2019). Use of Commercial Direct-to-Consumer Telemedicine by Children. Academic Pediatrics, 19(6), 665-669. https://doi.org/10.1016/j.acap.2018.11.016

Sardi, L., Idri, A. y Fernández-Alemán, J. L. (2017). A systematic review of gamification in e-Healthe-Health. Journal of Biomedical Informatics, 71, 31-48. https://doi.org/10.1016/j.jbi.2017.05.011

Sasangohar, F., Davis, E., Kash, B. A. y Shah, S. R. (2018). Remote patient monitoring and telemedicine in neonatal and pediatric settings: Scoping literature review. Journal of Medical Internet Research, 20(12), 1-9. https://doi.org/10.2196/jmir.9403

Tamayo, M., Besoaín, A. y Rebolledo, J. (2018). Determinantes sociales de la salud y discapacidad: actualizando el modelo de determinación. Gaceta Sanitaria, 32(1), 96-100. https://doi.org/10.1016/j.gaceta.2016.12.004

Vázquez, A., Jenaro, C., Flores, N., Bagnato, M. J., Pérez, M. C. y Cruz, M. (2018). -Health interventions for adult and aging population with intellectual disability: A review. Frontiers in Psychology, 9(NOV), 1-7. https://doi.org/10.3389/ fpsyg.2018.02323

Whitehead, M. y Dahlgren, G. (1991). What can be done about inequalities in health? Lancet, 26(338), 1059-1063. https://doi. org/10.1016/0140-6736(91)91911-D 